\title{
Extrasolar Trojan planets close to habitable zones
}

\author{
R. Dvorak, E. Pilat-Lohinger, R. Schwarz, and F. Freistetter
}

\author{
Universitätssternwarte Wien, Türkenschanzstr. 17, 1180 Wien, Austria \\ e-mail:dvorak@astro.univie.ac.at
}

Received 5 August 2004 / Accepted 10 September 2004

\begin{abstract}
We investigate the stability regions of hypothetical terrestrial planets around the Lagrangian equilibrium points $L_{4}$ and $L_{5}$ in some specific extrasolar planetary systems. The problem of their stability can be treated in the framework of the restricted three body problem where the host star and a massive Jupiter-like planet are the primary bodies and the terrestrial planet is regarded as being massless. From these theoretical investigations one cannot determine the extension of the stable zones around the equilibrium points. Using numerical experiments we determined their largeness for three test systems chosen from the table of the know extrasolar planets, where a giant planet is moving close to the so-called habitable zone around the host star in low eccentric orbits. The results show the dependence of the size and structure of this region, which shrinks significantly with the eccentricity of the known gas giant.
\end{abstract}

Key words. planets: Trojan planets - stars: individual: HD 114783 - stars: individual: HD 23079 - stars: individual: HD 4208

\section{Introduction}

When we are dealing with extrasolar planets and especially with terrestrial planets in habitable zones in such system we are aware that up to now we only have observational evidence for gas giants (=GG) like Jupiter. Therefore a dynamical stability study of possible additional planets of the size comparable to the Earth is a hypothetical one. It will be a task for future space programs to proof the existence of such planets.

There are different possible regions of motion in the so called habitable zones ${ }^{1}$ for such additional "terrestrial planets" which depend on the specific dynamical structure of the exoplanetary system. One can distinguish 4 different classes of such orbits:

1. $\mathrm{C} 1$ : when the $\mathrm{GG}$ is very close to the star there could exist such stable orbits outside the orbit of the GG for time scales long enough to develop a biosphere.

2. C2: when this GG moves far away from the central star (like Jupiter) then stable low eccentric orbits for additional planets can exist inside an orbit of the GG.

3. C3: when the GG itself moves in the habitable region a terrestrial like satellite (like e.g. Titan in the system of Saturn) could be in a stable orbit.

4. C4: when the GG itself moves in the habitable region a Trojan like terrestrial planet may move on a stable orbit around one of the Lagrangian equilibrium points $L_{4}$ or $L_{5}$.

1 A somewhat rough estimate for it is "where water could be in liquid form"; for a more precise definition see Kasting et al. (1993), therefore we concentrate our work on Trojan planets with a small initial eccentricity to minimise the difference of the radiation from the host star.
Besides the extensive study of Menou \& Tabachnik (2002) concerning the stability of orbits of terrestrial planets in extrasolar systems there exist investigations for specific systems for the classes C1 and C2: e.g. Eŕdi \& Pál (2003), Pál \& Sándor (2003), Dvorak et al. (2003a,b). In this new work we study the dynamical stability of possible terrestrial planets in the 1:1 resonance with the gas giant (class $\mathrm{C} 4$ ) for three selected known extrasolar planetary systems ${ }^{2}$. From the cosmogonical point of view one can imagine a possible formation of two planets in a 1:1 mean motion resonance (e.g. Laughlin \& Chambers 2002) as result of an interaction with the protoplanetary disc. They also investigated the stability of two massive planets in such a resonance which can be on stable orbits up to the masses of Saturn. In another study Nauenberg (2002) found an interesting stable configuration for motions in the 1:1 resonance, namely when the more massive planet has an almost circular orbit whereas the smaller body has a high eccentric orbit. Our interests were focused on terrestrial planets on low eccentric orbits in the 1:1 resonance, where only few studies have been undertaken like recently by Érdi \& Sándor (2004).

Out from the list of the approximately 120 planets in extrasolar systems compilated by Jean Schneider ${ }^{3}$ : we have chosen as test systems HD 114783, HD 23079 and HD 4208 (Table 1), where the presence of a gas giant was found which moves itself close to the habitable zone.

\footnotetext{
${ }^{2}$ We do not discuss the problem of habitable regions around a host star which is still somewhat in contradiction because it depends not only on the dynamical parameters of the orbits of a planet there, but also on the astrophysical parameters of the star like the spectral type and the age (e.g. Lammer et al. 2003)

3 The Extrasolar Planets Encyclopaedia at http://www.obspm.fr/encycl/encycl.html
} 
Table 1. Characteristics of the three exoplanetary systems with a giant planet moving close to the habitable zone.

\begin{tabular}{llcccc}
\hline \hline Name & $\begin{array}{l}\text { Spectral- } \\
\text { type }\end{array}$ & $\begin{array}{c}\text { Mass } \\
{\left[M_{\text {sun }}\right]}\end{array}$ & $\begin{array}{c}M \sin i \\
{\left[M_{\text {jup }}\right]}\end{array}$ & $\begin{array}{c}a \\
{[\mathrm{AU}]}\end{array}$ & Ecc. \\
\hline HD 114783 & K0 & 0.92 & 0.99 & 1.20 & 0.10 \\
HD 23079 & (F8)/G0V & 1.10 & 2.61 & 1.65 & 0.10 \\
HD 4208 & G5V & 0.93 & 0.80 & 1.67 & 0.05 \\
\hline
\end{tabular}

\section{Theoretical considerations}

In the model of the elliptic restricted problem there exist already many investigations concerning the stability of the Lagrangian points depending on the mass ratio of the primaries and the eccentricity of the orbit (e.g. Rabe 1967). Additional work has been done even for cases when the third mass is not regarded as massless (Marchal 1991). The results of a first order stability analysis in the framework of the general threebody-problem, loc.cit. p. 46ff) are presented there. With $M$ the total mass and $m_{1} \leq m_{2} \leq m_{3}$ a mass parameter $R$ was defined as $R=\left(m_{2}+m_{3}\right) / M+m_{2} \cdot m_{3} / m_{1}^{2}+O\left(m_{2}^{3} \cdot m_{3} / m_{1}^{4}\right)$. Using these results one can see that in the case of a terrestrial like planet with a relatively small mass compared to the two primary bodies there is practically no difference in the stability of the equilibrium points. When we take into account the observed eccentricity of the orbit of gas giant, furthermore the estimated (minimum) mass of the giant planet and a terrestrial planet with the mass of our Earth it turned out that almost all planetary systems (of the list given by Jean Schneider (loc.cit.) with a giant planet have Lagrangian points which are stable. But the extension of the stable region around this equilibrium points cannot be determined with such an analysis.

For the Jupiter Trojans the regions of motion have recently been determined via numerical integrations and also by mapping methods by different authors (e.g. Sándor \& Érdi 2003; Robutel 2004). In a simplified circular restricted problem these stability regions can be estimated via the Nekoroshev-theorem, where one finds that most of the known Trojans are in fact inside these stable regions. (Eftymiopoulos 2004). Unfortunately these methods cannot be applied for extrasolar systems because of the relatively large eccentricity. A detailed answer of the extension can be given only using the results of numerical simulations of each extrasolar planetary system under consideration.

\section{Numerical methods}

We have chosen two complementary numerical methods to answer the question of the largeness of the stability region; both use direct numerical integrations of the equations of motion.

\footnotetext{
- The LIE-integration method with an adaptive step size (Hanslmeier \& Dvorak 1984; Lichtenegger 1984). - A Bulirsch-Stoer integration.
}

As a first approach we started the computation in the dynamical model of the elliptic restricted problem consisting of the central star, the gas giant and a hypothetical (massless) terrestrial planet. The two primaries were always started in their periastron position; for the terrestrial planet we have taken the following grid of initial conditions: the different semimajor axis covered approximately $\pm 5 \%$ of the fixed semimajor axis of the gas giant with $\Delta a=0.01 \mathrm{AU}$. For the synodic longitude we have chosen the range from $20^{\circ}<\alpha<140^{\circ}$ with a gridsize of 0.2 degrees.

The method of analysis used was on one side the Fast Lyapunov Indicators (=FLI, Froeschlé et al. 1997) and on the other side the largest value of the eccentricity of the hypothetical Trojan planet during the integration time (Maximum Eccentricity Method = MEM). The integration time was in some cases up to $10^{6}$ years. Shorter integration times may show structures which disappear for longer time intervals of integration. The FLI is a well known chaos indicator; the MEM gives us the information of the evolution of the orbital parameters within the regarded time interval and reports also escapes from the region of motion. Via the eccentricity of the orbit the temperature difference on the surface of the hypothetical planet between periastron and apoastron of the terrestrial planet can be determined. For a stable climate on the hypothetical terrestrial planet which allows a stable biosphere to develop we estimate that $e<0.2$ during the integration time is a reasonable value. To ensure the validity of the results we always use the MEM and the FLI.

\section{Global results}

To take into account possible errors in the determination of the orbital eccentricity we have undertaken three different runs for three different eccentricities of the GG, namely the "observed" value \pm 0.05 . The initial eccentricity of the fictitious planet as well as the elements were set to zero. In the initial condition diagram, the maximum eccentricity of the fictitious Trojan planet during the integration time was plotted as a function of synodic longitude and initial semimajor axis. We can see in Fig. 1 how the extension of the "stable region" around the Lagrangian point for $e_{\mathrm{GG}}=0.05,0.10$ and 0.15 for the planetary system HD 23079 - as an example - shrinks rapidly with the primaries' eccentricity and almost disappears for $e_{\mathrm{GG}}=0.15$. The same behaviour can be observed for the other two systems which we investigated.

An interesting feature is that in the region close to the Lagrangian point itself the fictitious planet achieves the largest eccentricity. As example we show for HD 114783 for $e_{\mathrm{GG}}=$ 0.10 the curvature of the region with respect to the eccentricity (Fig. 4). This "hat" like structure appears in all regions around the points $L_{4}$ and $L_{5}$; the height of the "hat" depends on the mass of the GG and the eccentricity of its orbit. For an exact determination more computations are necessary.

\section{HD 23079}

HD 23079 is a central star of 1.1 Solar masses with a gas giant with a mass of 2.61 Jupiter masses which moves with a semimajor axis $a=1.65 \mathrm{AU}$ on an orbit with $e=0.1$ close to the habitable region. We varied the eccentricity of the gas giant in order to see how the structure of the stable region diminishes (see Fig. 1). For the lowest value of $e$ in the region of stable motion the eccentricity of the fictitious Trojan planet stays always 


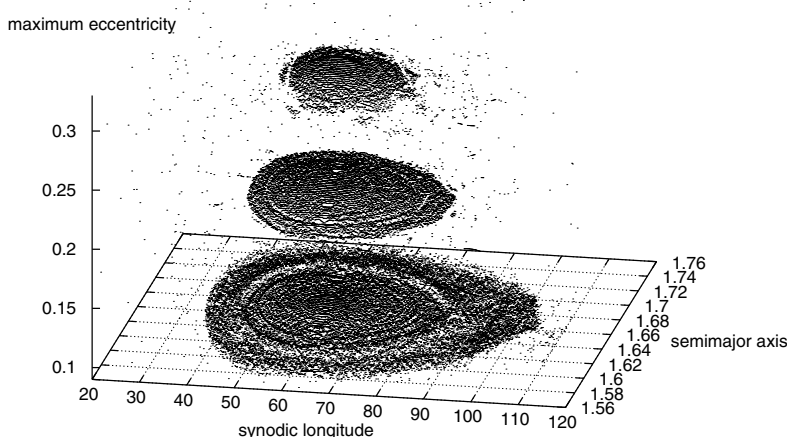

Fig. 1. Stability region around the system HD 23079 for three different values of the eccentricity $\left(e_{\mathrm{GG}}=0.05,0.1\right.$ and 0.15$)$ of the observed gas giant; for details see in the text.

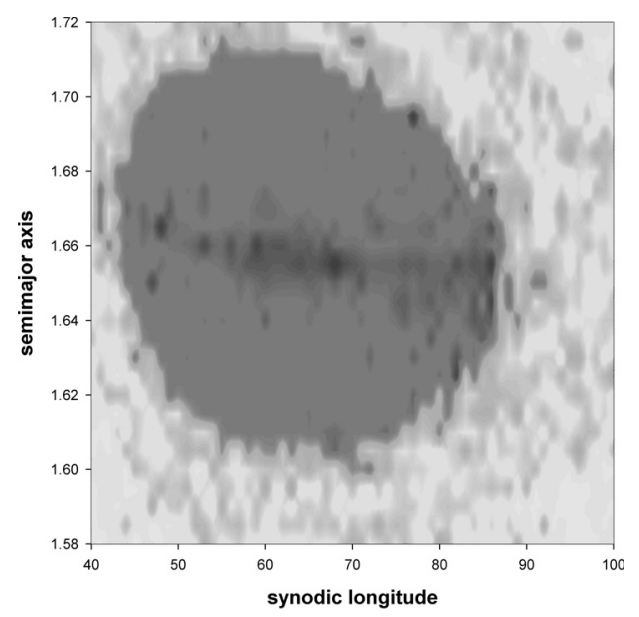

Fig. 2. FLIs for HD 23079 for $e_{\mathrm{GG}}=0.1$, dark regions indicate stable motion.

smaller than $e=0.1$, for the actual measured value of $e=0.1$ the eccentricity of the fictitious Trojan planet still fulfils the requirement mentioned above of always being below $e<0.2$; for a larger value $e=0.15$ the respective large eccentricities would - according to our hypothesis - not allow conditions for habitability.

We also determined the FLIs which show quite well the same behaviour as it was found with the MEM. There we also confirm the ring of less stable motion around the Lagrangian point (see Fig. 2). For the actual measured $e=0.1$ one can recognize a bar like structure which is not visible with the MEM method. On the contrary we can see different rings around the center (see middle graph of Fig. 1) by using the MEM. This structure is not present in the FLI plot.

This system was also investigated by another method, namely the RLIs (Relative Lyapunov Indicators) by Érdi \& Sándor (2004) for the actually determined eccentricity of the primary's orbit. A comparison of the respective results show the good agreement of all three results.

\section{HD 4208}

HD 4208 is a solar type star with 0.93 Solar masses; the gas giant with a mass of 0.8 Jupiter masses is orbiting the central

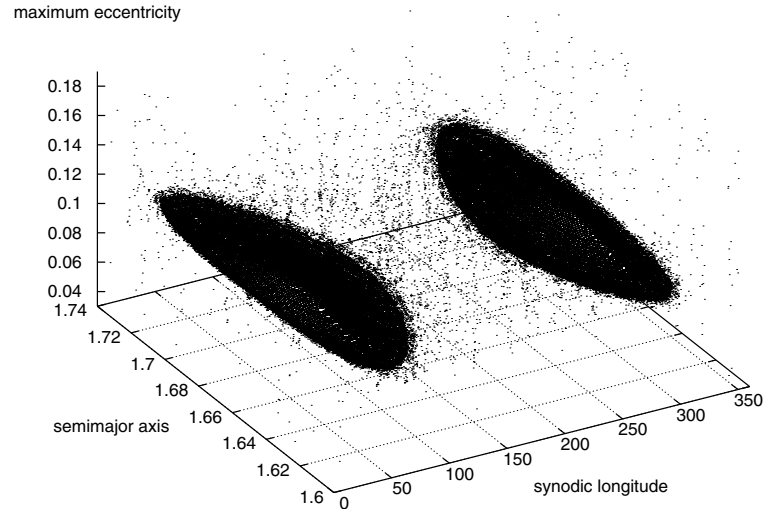

Fig. 3. Stability region around the system HD 4208; caption like in Fig. 1.

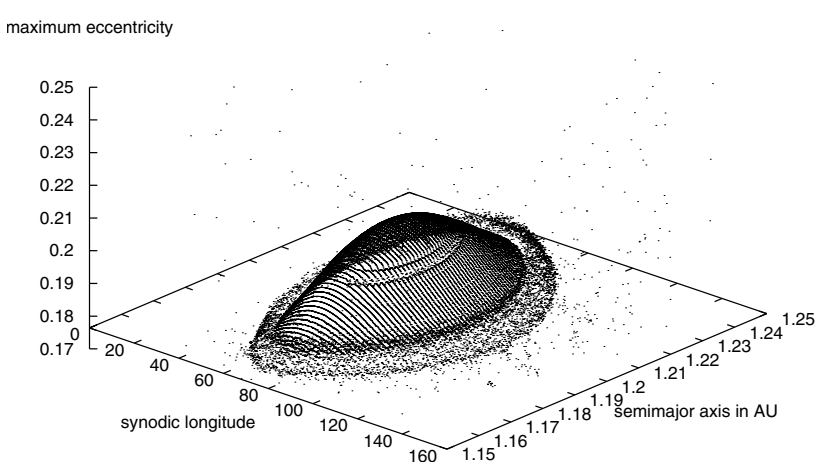

Fig. 4. Stability region around the system $\mathrm{HD} 114783$ for $e_{\mathrm{GG}}=0.1$; caption like in Fig. 1.

star with $a=1.67 \mathrm{AU}$ on an almost circular orbit $(e=0.05)$ which is always within the habitable zone. Again we varied this eccentricity to see how this changes the size of the stability region. For the actual value a large area around the Lagrangian point is stable and contains orbits for the fictitious Trojan planet which stays always close of being circular. For $e=0.1$ also orbits for "habitable" planets would be possible, for $e=0.15$ the large values of the eccentricity would lead the Trojan planet far out of the habitable zone in the apoastron and the periastron position. We also checked the region around $L_{5}$ which turned out to be of the same size as the other equilibrium point ${ }^{4}$. Furthermore the comparison with results achieved with the FLIs for the actual value of $e$ shows a quite good agreement.

\section{HD 114783}

HD 114783 is a K0 star with almost Solar mass (0.92) which hosts a gas giant of 0.99 Jupiter masses on an eccentric orbit ( $e=0.1$ ) with a semimajor axis $a=1.2$ AU. We show the results of the MEM in Fig. 4, where we can see the hat like structure with a maximum eccentricity of the orbits around $L_{4}$ close to the equilibrium point. The stable zone itself extends in the synodic longitude $30^{\circ} \leq \lambda \leq 110^{\circ}$ and in the semimajor axis from $1.16 \mathrm{AU} \leq a \leq 1.24 \mathrm{AU}$.

\footnotetext{
${ }^{4}$ There are about $1054 L_{4}$ Jupiter Trojans but only some $628 L_{5}$ Trojans, a fact which is not yet understood
} 


\section{Conclusions}

For all three extrasolar planetary systems investigated in this study there is a good chance for Trojan planets in the 1:1 resonance with the GG to stay on stable orbits with moderate eccentricities. This first stability study will be continued by a analysis of all possible exosolar systems with a giant planet in the habitable zone separately. The aim is then to find how the extension of the stable region around the equilibrium points depends on one hand on the eccentricity and on the other hand on the mass of the GG involved.

It may be that future observations of such systems using transits will provide quite interesting light curves (Schneider 2004) when one recognizes, that a Trojan has a very special orbit around the equilibrium point which consists of two well distinguished periods; for the Jupiter Trojans they are about 12 and 160 years.

Acknowledgements. R. Schwarz and F. Freistetter want to acknowledge the support by the Austrian FWF (Project P16024) and E. Pilat-Lohinger for the Hertha Firnberg Project T122.

\section{References}

Asghari, N., Broeg, C., Carone, L., et al. 2004, A\&A, 426, 353 Dvorak, R., Pilat-Lohinger, E., Funk, B., \& Freistetter, F. 2003a, A\&A, 398, L1
Dvorak, R., Pilat-Lohinger, E., Funk, B., \& Freistetter, F. 2003b, A\&A, 410, L13

Érdi, B., \& Pál, A. 2003, Dynamics of resonant exoplanetary systems, in Proceedings of the 3rd Austrian-Hungarian Workshop on Trojans and related topics, ed. F. Freistetter, R. Dvorak, \& B. Érdi, 3

Érdi, B., \& Sándor, Z. 2004, CMDA, in press

Efthymiopoulos, C. 2004, CMDA, in press

Froeschlé, C., Lega, E. \& Gonczi, R. 1997, CMDA, 25, 271

Hanslmeier, A., \& Dvorak, R. 1984, A\&A, 132, 203

Kasting, J. F., Whitmire, D. P., \& Reynolds, R. T. 1993, Icarus, 101, 108

Lammer, H., Dvorak, R., Pilat-Lohinger, E., et al. 2003, Atmosphere and orbital stability of exosolar planets orbiting gamma Cephei, in EGS-AGU-EUG Joint Assembly, Abstracts from the meeting held in Nice, France, 6-11 April 2003, abstract\#12443

Laughlin, G., \& Chambers, J. E. 2002, AJ, 124, 592

Lichtenegger, H. 1984, CMDA, 34, 357

Nauenberg, M. 2002, AJ, 124, 2332

Marchal, C. 1991, The Three-Body Problem (Elsevier)

Menou, K., \& Tabachnik, S. 2003, ApJ, 583, 473

Pál, A., \& Sándor, Zs. 2003, Dynamical stability of the habitable zones of exoplanetary systems, in Proceedings of the 3rd Austrian-Hungarian Workshop on Trojans and related topics, ed. F. Freistetter, R. Dvorak, \& B. Érdi, 25

Robutel, P. 2004, CMDA, submitted

Rabe, J. 1967, AJ, 72, 10

Sándor, Zs., \& Érdi, B. 2003, CMDA, 86, 301

Schneider, J. 2004, personal communication, COROT week 6, Paris 